\title{
IVF or ICSI for fertility preservation?
}

\author{
Bhorika Aggarwal', Amanda L Evans², Howard Ryan² and Sarah J Martins da Silva1 \\ ${ }_{1}^{1}$ Reproductive Medicine Research Group, School of Medicine, Ninewells Hospital and Medical School, University of Dundee, Dundee, UK \\ 2Human Fertilisation and Embryology Authority (HFEA), London, UK
}

Correspondence should be addressed to S J Martins da Silva: s.martinsdasilva@dundee.ac.uk

\section{Lay summary}

In IVF, eggs and sperm are added together for fertilisation to occur whereas ICSI involves injecting a single sperm into each egg. ICSI is very effective where sperm count or swimming is poor (male infertility) but is slightly riskier than IVF in terms of health problems in children, although these risks are small. However, the risk of no eggs fertilising is higher for IVF compared to ICSI and couples undertaking fertility preservation, for example, before cancer treatment, usually only have time for one attempt. Using fertility preservation treatment cycle data reported to Human Fertilisation and Embryology Authority (HFEA), this study shows that ICSI results in higher number of fertilised eggs and embryos for storage or treatment compared to IVF. However, 19\% of eggs are not used in ICSI treatment, so IVF appears to be better overall. Clinics should choose IVF or ICSI for fertility preservation depending on sperm characteristics rather than using ICSI for all.

Intracytoplasmic sperm injection (ICSI) is highly effective for male factor infertility. However, its use for non-male factor infertility has increased dramatically worldwide in the last 2 decades despite little evidence demonstrating effectiveness in this population. The rationale for using ICSI is to reduce the risk of low or total failed fertilisation (TFF), thereby increasing the number of embryos and the potential for pregnancy and live birth (Bhattacharya et al. 2013). A meta-analysis (Johnson et al. 2013) of sibling oocyte studies reported a significantly higher pooled relative risk of TFF with IVF compared to ICSI. In contrast, more recent studies of infertile couples with non-male factor infertility show no difference in fertilisation, implantation or pregnancy rates (Li et al. 2018), even in poor responder patients (Sfontouris et al. 2015) or advanced maternal age (Tannus et al. 2017).

Arguing against an approach of ICSI for all there is accumulating information on the health of offspring including, amongst others, increased risk of congenital malformations, chromosomal abnormalities and epigenetic syndromes compared to naturally conceived children (Davies et al. 2017, Xiong et al. 2017, Esteves et al.
2018) and lower sperm concentration in male offspring (Belva et al. 2019).

Overall, TFF has been reported to complicate 1-3\% ICSI and 5-8\% IVF cycles (Swain \& Pool 2008). This is particularly relevant for couples undertaking emergency fertility preservation who may only have one opportunity to create embryos. As such, there is a genuine debate regarding the correct approach to fertilisation for this particular group of patients: whether to apply IVF or ICSI depending on sperm characteristics or to undertake ICSI for all. In an attempt to resolve this dilemma, we analysed data provided by Human Fertilisation and Embryo Authority (HFEA). We present data for UK fertility preservation cycles 2015-2018 and 218,830 oocytes retrieved (Table 1), with known insemination method, fertilisation and downstream embryo disposal (transferred, stored, donated). Fertilisation rate (FR) was calculated from the number of oocytes normally fertilised (2PN) divided by the number of inseminated oocytes (IVF) or the number of oocytes microinjected (ICSI).

In total, 75,350 eggs were inseminated (IVF) and 108,901 eggs were injected (ICSI). FR was significantly higher for ICSI compared to IVF $(72.8 \%$ vs $64.9 \%$; 
conventional in vitro fertilization compared to intracytoplasmic sperm injection in Bologna poor responders with a single oocyte retrieved. Journal of Assisted Reproduction and Genetics 32 691-697. (https://doi.org/10.1007/s10815-015-0459-5)

Swain JE \& Pool TB 2008 ART failure: oocyte contributions to unsuccessful fertilization. Human Reproduction Update 14 431-446. (https://doi.org/10.1093/humupd/dmn025)

Tannus S, Son WY, Gilman A, Younes G, Shavit T \& Dahan MH 2017 The role of intracytoplasmic sperm injection in non-male factor infertility in advanced maternal age. Human Reproduction $\mathbf{3 2}$ 119-124. (https://doi.org/10.1093/humrep/dew298)
Xiong X, Dickey RP, Buekens P, Shaffer JG \& Pridjian G 2017 Use of intracytoplasmic sperm injection and birth outcomes in women conceiving through in vitro fertilization. Paediatric and Perinatal Epidemiology 31 108-115. (https://doi.org/10.1111/ppe.12339)

Received in final form 22 December 2020

Accepted 11 February 2021

Accepted Manuscript published online 11 February 2021 (c) 2021 The authors Published by Bioscientifica Ltd
This work is licensed under a Creative Commons Attribution 4.0 International License.

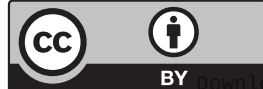

aded from Bioscientifica.com at 04/26/2023 01:38:06PM via free access 